\section{Biotech basic training}

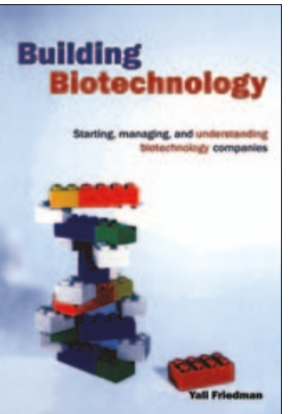

\section{Building Biotechnology: Starting, Managing, and Understanding Biotechnology Companies}

by Yali Friedman

thinkBiotech, 2004

264 pp. hardcover, $\$ 34.95$

ISBN 0-9734676-2-2

\section{Reviewed by Aris Persidis}

Biotechnology is widely acknowledged to be a great industry in which 을 to work, for its combination of social benefits and potential financial rewards. It is constantly in the news - for both good reasons and bad-and is now mature enough in the public markets to merit its own indices and a legion of analysts that track stock performance for over 400 publicly traded companies. At the same time, biotechnology is perceived to be a strategic industry for many countries, states and regions because of its potential for high-tech job creation and competitive advantages in trade. Graduates from life science programs around the world now readily consider careers in biotechnology, and because of significant recent mergers and acquisitions among companies of all sizes, there is a pool of biotech workers who are often on the move. In addition, there are those in the biotech-related legal and financial industries, armchair observers and pundits, all of whom are fascinated by the biotech industry. It is to all these groups that Yali Friedman addresses his book, Building Biotechnology.

The need for a comprehensive guide to understanding the inner workings of corporate biotechnology is very acute, because there are so many different elements to building a company in this space. They include intellectual property (IP), regulatory constraints, raising capital, attracting personnel, managing $\mathrm{R} \& \mathrm{D}$, manufacturing, marketing, outsourcing, services, as well as the actual science and technology itself. Each of these areas is the subject of entire semester courses at business school, but what if we want one comprehensive source? This book does much to fulfill that role.

The book is divided into four main sections. Friedman sets the stage by highlighting the necessary interplay between the legal, regulatory, business and science factors that affect biotechnology. This is a significant point, and if not properly grasped, often leads to misunderstandings or views that are heavily biased. In order to practice biotechnology or to understand an event one reads about in a newspaper, it is critical to be aware that science and technology are but the beginning. Before a drug succeeds or fails in clinical trials, it has been repeatedly tested

Aris Persidis is at Upstate Group, 706 Forest Street, Suite 1, Charlottesville, Virginia 22903, USA.

e-mail:apersidis@upstate.com in multiple nonhuman settings and has been heavily regulated. Its IP issues - patents, know-how and trade secrets-have been addressed, and financial support of hundreds of millions of dollars over a decade or so have been invested. This section of the book also includes a useful chronology and description of some of the most noteworthy events that helped shape the industry, including major discoveries, legislation that enabled technology transfer and therefore the birth of corporate biotechnology as we know it today, and the establishment of pioneer companies such as Genentech, Genzyme, Amgen, Chiron, Celera and others.

The next section focuses entirely on IP and regulatory issues. IP is often thought of as a 'black art' because of ever-changing laws and judicial decisions, and the difficulties of working in the field are compounded by the fact that the US, Europe and Japan treat patent issues differently. This is a section where the reader may want to invest some time. Beginning with basic principles, such as filing for a patent before you publish or present at a conference-useful advice for academic researchers who have commercial aspirations - the section delves into a number of key issues and subtleties. Regulatory issues are also well represented. The stages of clinical trials, as well as issues with generic manufacturers are included, as are regulations affecting plant crops and genetically modified organisms. Although providing data only for the US Food and Drug Administration and not for its European or Japanese counterparts (the interested reader would benefit from knowledge of the differences), this section is a useful primer of regulatory issues.

The third section, on the business realities of corporate biotechnology, answers some fundamental questions: How does one set up a biotech company? Where does the money come from? What business models are there? How are the research and marketing functions managed? Other relevant issues are addressed, including failure itself - especially a product that fails in the clinic. Given the high rate of attrition that products have at late stages of development, failure management strategies are as critical as any other strategy. Details about writing business plans are not covered in this book, but a discussion about the central importance of quality management is included, and rightly so.

The final section is a basic introduction of major areas of science and technology. There is such an abundance of information and history in the industry that it is impossible for any concise effort to cover more than the surface, and readers should not expect to receive the broadest or deepest education here. Nevertheless, it succeeds in acquainting those unfamiliar with the fundamentals of molecular biology, cell biology, drug development and screening, as well as well-known applications and technologies.

Overall, Yali Friedman's coverage of the subject matter is impressive in scope. The book is well suited for novices, for classroom use and interested readers from outside the industry. It will also serve active practitioners as a good collection of all the chapter headings they need to be aware of, and sometimes forget. In this, it complements other well-known books (cited in the appendix) that cover biotechnology from the human perspective and focus on the stories that have made this industry great. 